\title{
Natural compounds extracted from Moringa oleifera and their agricultural applications
}

\begin{abstract}
Natural bio-active compounds synthesized by plants as secondary metabolites are well known and established. Today, their application in various fields such as medicine in the form of drugs and biopesticides in agriculture is well documented. In recent times, the delivery of such compounds is achieved through nanodelivery technology, which is gaining acceptability in both field of drugs and agrochemical industries. The bio-active compounds with chemical diversity are obtained from nature either as homogenous plant crude extracts or as purified compounds. Crude plant extracts exist as a combination of different bio-active compounds with various polarities, and their partition remains a challenge in the process of characterization and identification. Extraction of these compounds from plant species is achieved by different solvents and extraction methods. Analytical methods like HPLC have commonly been utilized with GC-MS and LC-MS/MS chromatography methods to identify the compounds. Crude extracts from different morphological parts of plant species including Moringa oleifera are increasingly becoming important in the context of agricultural pest management and human medicine. M. oleifera is a medicinal plant that synthesizes such metabolites which include phenolic acids, carotenoids, quinones, antraquinones, flavonoids, flavonols, flavones, tannins, alkaloids, coumarins, terpenoids, amines, cyanogenic glycosides, triterpenoids, non-protein amino acids, glucosinolates, polyacetylenes, polyketides, phenylpropanes, steroids and saponins. They exert biological activities and can potentially be used to retard microbial activities. Other uses of $\mathrm{M}$. oleifera are medicinal uses and other purposes such as water purification, fertilizer, biogas and biopesticides. The aim of this chapter is to highlight the uses and profiling of bio-active compounds of M. oleifera, their mode of action and prospects in commercial biopesticides for agricultural applications.
\end{abstract}

Keyword: Bio-active compounds; GC-MS; M. oleifera; Mode of action; Nanotechnology 